\title{
Design Opportunities for Wearable Devices in Learning to Climb
}

\author{
Eleonora Mencarini ${ }^{1 \& 2}$, Chiara Leonardi ${ }^{1}$, Antonella De Angeli ${ }^{2}$, Massimo Zancanaro ${ }^{1}$ \\ ${ }^{1}$ Fondazione Bruno Kessler (FBK) \\ Trento, Italy \\ \{mencarini, cleonardi, zancana\}@fbk.eu \\ ${ }^{2}$ University of Trento \\ Trento, Italy \\ antonella.deangeli@unitn.it
}

\begin{abstract}
In this paper, we present a field study on the learning of climbing aimed at defining the design space of wearable devices to support beginners. Three main findings have emerged from our study. First, climbing has a strong emotional impact on beginners; therefore, learning to climb requires mastering new motor patterns as well as negative emotions, such as stress and fear. Second, the feeling of danger that climbers often experience can be mitigated by trust in the climbing partner and the perception of her active presence. Finally, a big problem in climbing is the communication difficulty between the climbing partners and between climber and instructor. We conclude the paper presenting four design considerations for the design of wearable devices meant to support the learning of climbing by providing the actors involved with augmented communication. Such augmented communication should address both the physical and the emotional difficulties of this sport.
\end{abstract}

\section{Author Keywords}

Climbing; emotions; wearables; augmented communication.

\section{ACM Classification Keywords}

H.5.2. User interfaces: User-centered design.

\section{INTRODUCTION}

In recent years, the deployment of wearable devices in the sport domain has gained increasing popularity both in the market and in HCI research. Several bracelets for monitoring physiologic functions (such as heartbeat, sleep quality, etc.) and for keeping track of workouts have been launched on the market, e.g. the Fitbit, the Jawbone UP, and the Garmin vìvo series. Similarly, part of the research in HCI in sports has focused on supporting training through wearable devices by keeping track of physical performance, e.g. $[14,20]$, and by providing real-time feedback, e.g. [1,13].

\footnotetext{
Permission to make digital or hard copies of all or part of this work for personal or classroom use is granted without fee provided that copies are not made or distributed for profit or commercial advantage and that copies bear this notice and the full citation on the first page. Copyrights for components of this work owned by others than the author(s) must be honored. Abstracting with credit is permitted. To copy otherwise, or republish, to post on servers or to redistribute to lists, requires prior specific permission and/or a fee. Request permissions from Permissions@acm.org.

NordiCHI'16, October $23-27,2016$, Gothenburg, Sweden Copyright is held by the owner/author(s). Publication rights licensed to ACM.

ACM 978-1-4503-4763-1/16/10..\$15.00

DOI: http://dx.doi.org/10.1145/2971485.2971509
}

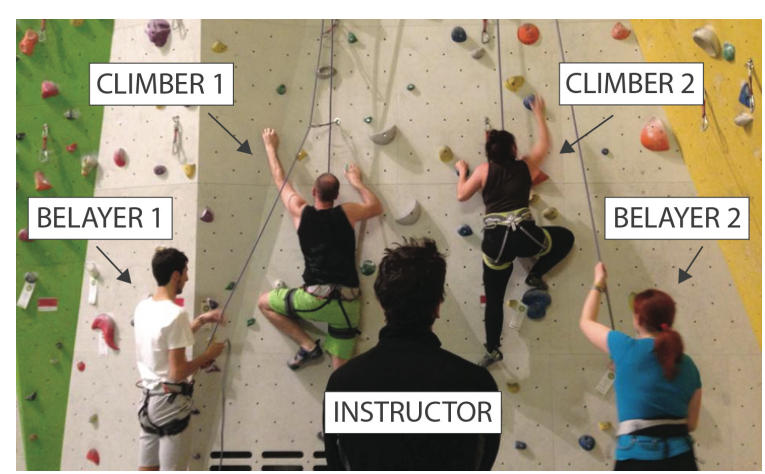

Figure 1. A climbing lesson.

A part of these studies focused on the initial phase of learning a new sport in order to facilitate the acquisition of new motor skills $[10,21,25]$. However, the learning of a new sport often entails a high level of emotional involvement, which may influence the performance [9]. In particular, the negative emotions due to the lack of familiarity with a new activity can affect the development of new motor skills [26]. So far, only a few studies in HCI have addressed the impact of emotions in sport performance. Among those, a notable example is [28], which investigates the effect of remote cheering up on runners during long races.

Our research question is whether and how wearable devices can support the learning of climbing, a sport that entails a great physical and emotional involvement. Climbing is becoming more and more popular thanks to the increasing number of indoor gyms and the improved safety [7]; nevertheless, it is still considered an extreme sport. Climbers tackle the rock with bare hands, employing their body as a tool and moving up with strength, precision, and search for balance. The equipment they use is meant just for ensuring safety; the only artefact meant to support performance are the climbing shoes. Considering the minimalistic approach to the equipment and the centrality of the body peculiar to this sport, we decided to investigate the design of wearable devices since they are a portable and unobtrusive technology.

This paper offers an original contribution to the design of wearable devices for the learning of climbing, considering them as tools for the augmentation of the communication among the actors involved (fig.1). In this way, both the motor and the emotional aspects implicated in the learning of this sport can be addressed and managed. We provide four design 
considerations based on a rich field study and focus on what kind of support wearable devices can offer when negative emotions take over. In our view, the goal of the design should not be to eliminate negative emotions, but rather to help actors managing them, while considering the difficult balance between confidence and fear typical of climbing. Finally, we provide some reflections on the potential design explorations that the adoption of wearable devices as Augmented Communication devices can offer.

The paper is organized as follows. In the next section, we provide an overview of the most relevant research in related fields; in section 3, we describe the methodology adopted; in section 4 , we present four main themes that characterize the experience of learning how to climb. In section 5 , we present the design considerations and in section 6 , we discuss some possible concepts. In section 7, we list the limitations of this work and the goals of future ones. In the last section, we present the conclusions.

\section{RELATED WORK}

\section{$\mathrm{HCl}$ and the Learning of a New Sport}

The term motor learning describes a lasting change of motor performance caused by training, and it can be divided in three stages: cognitive, associative, and autonomous [22]. In the cognitive stage, the coach gives explanations about how to perform a new movement and the trainee tries to understand what is required. In the associative stage, the trainee tries to match the correct movement with her internal sensations, and progressively improve by trial and error. To know whether she is performing well, she needs to match her intrinsic feedback with an extrinsic feedback. The intrinsic feedback is the one that originates from the trainee's perception of her own movements and position in space (i.e. the feeling of proprioception), while the extrinsic feedback is the one that comes from an external agent, such as the coach [22]. Thus, the presence of an extrinsic feedback is very important in order to understand the correctness of the proprioceptive sensations that the body feels [22]. Finally, in the autonomous stage, the trainee should have internalized the right movement and should not need guidance anymore.

Despite the role of the coach is so important in the first two stages of learning, the direct contact between trainer and trainee is not always possible. In several sports, e.g. climbing, surfing, and parachuting, distance between trainer and trainee may hinder vision, audio, and the implementation of the usual teaching techniques, like imitation and direct modification of the trainee's body posture. For this reason, part of HCI research in sports has addressed the topic of learning mostly exploring augmented feedback through different sensory modalities. We report here two examples of studies that compared augmented feedback in the audio, visual and haptic modalities. Bächling et al. [1] compared audio, visual, and tactile feedback for the training of swimmers and showed that audio is the modality that performed worst requiring the longest time of reaction to the athletes. Similarly, Kosmalla et al. [13] compared the three modalities in a climbing setting and showed that the visual modality performed worst. These two studies show that the suitability of augmented feedback is strongly influenced by the kind of sport and by the environment in which it is performed.

\section{The Climbing Experience}

Some researchers have investigated the motivation and the experience of climbers. MacAloon and Csikszentmihalyi [18] describe climbing as one of the sports that entails the highest levels of flow. With the term flow, the authors describe those moments when somebody is completely focused and in control of what she is doing, with no external thought or emotion to interfere, and finding pleasure in the activity itself, regardless of the results. Being in a state of flow is not an objective condition, but it depends on the subjective perception of the challenges that an activity provides; there has to be a perceived challenges-skills balance. Climbing can be a great source of flow because there are several levels of difficulty, among which every climber can choose the challenge that better matches her skills, and it requires a deep focus, which makes climbers be totally absorbed in the activity and isolated from the rest. In his progressive model of the motivation for climbing, Levenhagen [15] puts flow at the initial stage, followed by the building of character and the realization of a greater spiritual self. The first is intended as the demonstration to the self and to the others of the climber's value by engaging in more and more difficult climbs, while the latter refers to the creation of a meaningful world by using her will to tame it and deliberately deciding to risk her life. Similarly, Lewis [16] underlines the unmediated relationship with the rock through the physical and tactile engagement, and the achievement of a sense of freedom that comes from the sense of human embodied agency on the environment.

\section{Wearable Technology for Climbing}

Currently, technologies for climbing can be grouped in two main clusters: augmented walls and wearable devices. The majority of works explore the augmentation of walls; nevertheless, in this paper we focus on wearable devices. Wearable devices for climbing are usually designed for expert sportsmen who want to keep track of their performance. For example, Ladha et al. [14] designed and evaluated ClimbAX, a wristband for automatic skills assessment that is able to detect power, control, stability, and speed in climbing sessions. Similarly, Kosmalla et al. [12] designed a wrist-worn device capable of recognizing the routes climbed in a gym and thus allowing the comparison of training sessions between climbers. Looking at outdoor climbers, Schöning et al. [23] proposed a concept design based on Location-Based Systems and Augmented Reality embedded in the climbing gear addressing some of the main problems of mountaineering such as location finding, communicating with the climbing partner, and knowing weather conditions. 
The two different contexts of practice, indoors and outdoors, have been demonstrated to have a strong influence on the climbers' attitude towards wearable technology. Daiber et al. [4] investigated the climbers' acceptance of wearable devices aimed at recording data during workout sessions and found that it changes according to their motivation for climbing. While outdoors nature-lovers climb for fun and recreation and are not interested in data about their performance, indoor-training enthusiasts are keen on performance and competitiveness, and would be interested in monitoring themselves.

\section{METHODOLOGY}

In this paper, we focus on sport climbing. Unlike other kinds of climbing such as ice climbing, free solo, bouldering, etc., sport climbing can be practiced either indoors on artificial walls, or outdoors on the rock. It involves two people: a climber and a belayer (fig. 2) and trainees have to acquire the skills of both roles. As climbers, they have to learn to ascend a route leading (i.e. going first), and in top-rope (i.e. going after the first); and as belayers, they have to learn how to handle the rope properly by means of a friction device in order to hold a possible climber's fall. There is a great difference between leading and climbing in top-rope in terms of risks and tension involved. When leading, the climber brings up the rope tightened to her harness and has to clip it in the quickdraws in order to protect herself. In case of fall, the leading climber will fall until her last protection, while, when climbing in top-rope, the rope comes from above and, in case of fall, she will fall just for the elasticity of the rope.
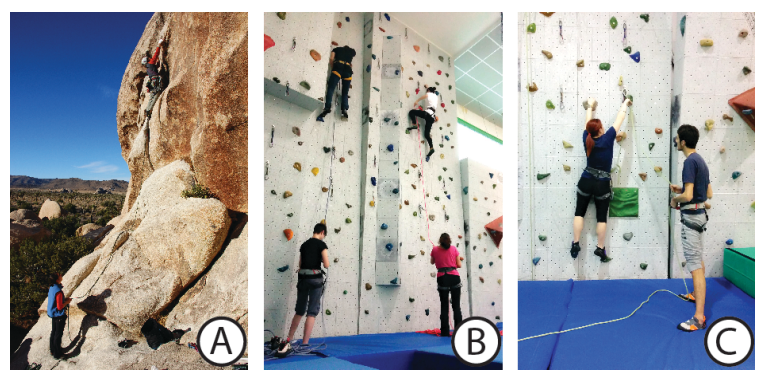

Figure 2. A) Two partners climbing outdoors; B) Two pairs climbing indoors; C) A leading climber clipping the rope.

When it is not learnt by following friends already practicing it, climbing is usually taught in courses held by qualified instructors. During the lesson, the trainees work in pairs and alternate the role of climber and the one of belayer. The instructor usually follows the climber by standing on the ground next to the belayer, so that she can intervene in case of need (fig. 1).

\section{Field study}

In order to explore the issues that beginner climbers face when they start to learn this sport, we conducted a series of observations of a course in a climbing gym and semistructured interviews with both trainees and mountain guides. For a summary of each phase of the field study and relative goals, see Table 1 .

\begin{tabular}{|c|c|}
\hline Fieldwork & Dimensions investigated \\
\hline $\begin{array}{l}\text { Contextual } \\
\text { observations of } 6 \\
\text { indoor climbing } \\
\text { lessons }\end{array}$ & $\begin{array}{l}\text { - } \quad \text { Teaching strategies } \\
\text { - } \quad \text { Communication between instructors and } \\
\text { trainees, and between climbing partners } \\
\text { - Trainees' difficulties }\end{array}$ \\
\hline $\begin{array}{l}\text { Semi-structured } \\
\text { interviews with } \\
11 \text { trainees }\end{array}$ & $\begin{array}{l}\text { - } \quad \text { Motivation for taking a climbing course } \\
\text { - } \quad \text { Mifficulties encountered } \\
\text { - } \quad \text { during the different phases of the activity } \\
\text { - } \quad \text { pommunication between climbing } \\
\text { - } \quad \text { Risk-taking attitude } \\
\text { Trust }\end{array}$ \\
\hline $\begin{array}{l}\text { Semi-structured } \\
\text { interviews with } 6 \\
\text { mountain guides }\end{array}$ & $\begin{array}{ll}\text { - } & \text { Teaching strategies } \\
\text { - } & \text { Course objectives and content } \\
\text { - } & \text { Learners' most frequent errors }\end{array}$ \\
\hline
\end{tabular}

Table 1. Summary of the phases of the field study.

\section{Observations}

The observations were conducted during 6 indoor lessons with 9 trainees ( 4 males, 5 females, aged $M=26$ years) and one mountain guide as the instructor. Each observation lasted for the duration of the lesson, i.e. 2 hours. Notes (with a particular focus on dialogues) and pictures were taken. Given the high risk of falls and injuries inherent to climbing, we opted for shadowing observations and limited the questions to the instructor and to the learners during the activity. The purpose of the observations was to understand how climbing is taught in real contexts, with a special regard to the learners' main difficulties, the teaching strategies, and the communication among the people involved in the activity.

\section{Interviews to the trainees}

We conducted semi-structured interviews with 11 trainees; 7 of them were attending the indoor course we observed, while the other 4 were attending a rock-climbing course, which was more focused on outdoor climbing. The participants were 4 males and 7 females (aged $M=24$ years), with a high variability in climbing experience $(M=21$ months, $S D=27.8$ months). We conducted the interviews individually and each of them lasted approximately 30 minutes. The main purpose of these interviews was to investigate trainees' motivation, objectives, main difficulties, and the emotional aspects of climbing.

\section{Interviews to the mountain guides}

Finally, we interviewed 6 male mountain guides (aged $M=$ 37 years; years of work as mountain guide $M=8$ ). One of them was the instructor of the indoor course where we conducted the observations, while the others were recruited among personal contacts. Each interview lasted approximately one hour. These interviews aimed at understanding the goals, the content, and the structure of a climbing course, guides' teaching strategies as well as what they like the most in climbing and in their job, and the issues they encounter most frequently. 


\section{Data Analysis}

The analysis started when the data were still being collected and has been an on-going process for 6 months. This continuous process allowed us to refine the questions and directions of the investigation as part of the field study. The observations worked as initial exploration of the situated practices of learning and teaching climbing. We could understand how a climbing course is organized, and what the main difficulties of trainees and the main teaching strategies are. Pictures (approximately 150) and notes (about 35 pages) were taken during the observations. The interviews to the trainees and to the guides resulted in 10 hours of audio recording. All the notes from the observations and the audio recordings of the interviews were analyzed using the software Atlas.ti. Firstly, we analyzed the observations through the lens of Contextual Inquiry [2] in order to identify the elements that characterize the situated practice of learning climbing; secondly, the interviews were analyzed through Grounded Theory [8]. Grounded Theory was first used to elicit and compare emergent themes in order to identify and refine interpretative categories. In a second phase, we compared the identified categories with the literature in order to explain the dynamics of the case study. In the following section, we report the findings of our data analysis in the form of themes, which emerged across both the observations and the interviews. These themes highlight the challenges that the emotions involved in the learning and teaching of climbing offer to the design of technology. Quotations from the interviews are reported in anonymous form: mountain guides will be quoted using MG1, MG2, MG3, etc., while trainees will be quoted as T1, T2, T3, etc.

\section{THEMES}

We identified four pivotal themes for understanding the practices and the experience of learning how to climb, namely: i) Positive emotions, ii) Negative emotions, iii) Trust, and iv) Communication.

\section{Positive Emotions}

Climbers do not primarily seek for adrenaline or for competitiveness - these are just some of the components that characterize this sport - rather, they seek for the positive feelings it can provide. The main sources of gratification in this sport are the sense of self-efficacy, the flow, and the sense of adventure. These reflections have been reported mostly by mountain guides, probably because they already master this sport, but we had some hints also from beginners.

\section{Self-efficacy}

Except for the shoes, sport climbing does not involve any support tool for the activity. The gear used is meant to ensure the climber's safety, but the rock wall is tackled just with the body, through the skillful use of strength and movement techniques. The direct relation with the rock and the use of the body as a tool are revealed by T1's words when she states, "the beautiful thing, in my opinion, is that you feel you go up thanks to your body, to your limbs; you do things with your hands, you perceive that it's you who is doing that". For this sense of agency, every ascent is a challenge with yourself and it can bring a great sense of satisfaction and self-efficacy when you are able to overcome the difficulties you encounter, but also of frustration, if you fail.

\section{Flow}

In accordance with what has already been mentioned in the previous literature [18], the concept of flow emerged strongly also in our field study. Climbing can be a great source of flow because there are several levels of difficulty among which every climber can choose the challenge that better matches her skills. Moreover, it requires a deep focus which makes climbers totally absorbed in the activity and isolated from the rest. In the interviews, the mountain guides reported a few examples that highlighted the experience of flow in climbing. For instance, they mentioned that the high level of focus that climbing requires works as liberation of the mind, e.g. "You cannot even think of something else, it's necessarily liberating" MG3. Moreover, some of them reported that they feel a great sense of satisfaction when they are totally absorbed in the activity and isolated from the rest. As MG2 stated "[When I climb] I'm at home, I feel at ease, and the world doesn't exist anymore; only the 4sqm where I'm moving exist, [and they appear] as I move along them. In this sense, climbing is liberating. It is my corner of paradise. I feel good when I climb even if I snort because it's difficult".

\section{Adventure}

Most of the people we interviewed did not consider climbing just as a sport, but as an adventurous outdoor experience. Indeed, usually indoor gyms are meant as training places for then going climbing outdoors, on the rock. The ultimate goal of many climbers is to climb on multi-pitch routes in the mountains because there they can find the highest level of self-challenge and adventure. As T3 affirmed, "I'm trying to learn the basics so that then I can go climbing outdoors. The outdoors is the achievement of a goal, there you can arrive to the top and see all the mountains around have to offer." The guides reflected upon this as well: "Climbing is beautiful when practiced outdoors, in a natural environment. There it can entail its adventurous character" MG5. Moreover, climbing outdoors is seen as a sort of a privilege since it enables you to discover new places, accessible to few people "Climbing is exploring, going up to see what is beyond" MG5.

\section{Negative Emotions}

While positive emotions become part of the practice when climbers master at least the basics, during the learning, beginners often experience a series of negative emotions caused by their lack of motors skills and by the novelty of the vertical movement. The most common negative emotions are discomfort, stress, fear, and panic, and they can be seen as a continuum of increasing intensity. In the following paragraphs, we will discuss the learning difficulties that discomfort and fear provide and the coping strategies instructors adopt. 
The Discomfort of the Vertical Movement

At the beginning, climbing is mostly an emotional experience. Since vertical movement is not something that people deal with in daily life, learners experience a general feeling of uneasiness due to their clumsiness. MG2 made an interesting comparison when he affirmed, "this is exactly what happens to a child who is learning to walk. Why is she clumsy? Because she has to learn the sensations (...) and the beginner climber is exactly in the same situation, even psychologically because she feels unprotected, not because climbing is scary in itself, but because she is not able to recognize the sensations she feels".

From the observations and the interviews with the guides, it emerged that usually beginners try to reach the top of a climb as quick as possible in order to overcome their discomfort. Climbing, on the contrary, is about efficient and fluid movements, as shown from this short dialogue between instructor and trainee: MG1: "C., what's your goal [for this ascent]?" C.: "Getting to the top...?" MG1: "Getting to the top is a trivial goal!" Indeed, a large part of the training is dedicated to teaching the basics of climbing movements, such as shifting the weight on the thrusting leg before starting to move, pushing with the legs instead of pulling with the arms, finding resting positions, etc. During the interviews, the trainees confirmed those to be the most challenging movements and explained that the difficulty of learning not only lies in the acquisition of the right movement sequences, but also in the effort to overcome the fear that the execution of these movements entails ("When there are difficult moves to do, I become demoralized and I never try" T8). Therefore, movement and emotional states are strictly intertwined in climbing; one influences the other.

\section{Coping Strategies}

Moving away from the usual motor schemes is psychologically demanding; beginners need to learn to feel confident in the vertical dimension. Therefore, the very first objective of a climbing course is to make trainees familiarize with verticality. In order to do this, instructors foster firsthand experiences, and adopt special techniques and auxiliary artifacts to make trainees learn through direct body sensations. For instance, in the lessons, the guide asked trainees to use an auxiliary rope to clip in the quickdraws when climbing top-rope in order to make them feel the changes of balance that will occur when clipping as leading climbers. Furthermore, in the interviews, the guides affirmed that sometimes they try to make trainees visualize implicit or hidden mechanisms, e.g. visualizing the shift of the center of gravity while walking with the help of a hanging weight tied to the harness. The general purpose behind this kind of exercises is to develop proprioception, i.e. kinesthetic awareness. Indeed, every exercise is usually followed by the instructor's investigation of the trainee's personal sensations "How was it? How did it feel? It is better this way, isn't it?" MG5. Some other exercises, such as having the climber wearing sneakers on the hands, aim at limiting the grasping instinct, while keeping a hand behind their back aims at intensifying sensorial perceptions through body constraints. By putting the trainees in a state of need, the guides help them to focus on how they move, find balance, and gain selfconfidence.

\section{Fear}

The feeling of clumsiness that beginners experience can rapidly turn into fear because, in addition to the novelty of the movement patterns, the vertical dimension implies objective danger. During the interviews, several trainees agreed that fear is the prevalent emotion while learning how to climb ("Fear is the first that you feel" T8). They fear injuries, heights, falls, and handling gear. The latter is a great source of stress for both climbers and belayers because the safety of the activity depends on the right management of gear. Looking deeper at emotions, we saw that beginners perceive climbing (especially when leading) as an alternation of fear and relief. As the trainee T8 explained, "Leading... focused, yes, a lot, it takes me longer to think of the next move and especially whether [holds] are good enough; scared also a lot, then safe when I clip the rope in the quickdraws, in that moment I feel... relieved; then, when I move again from the quickdraw on, I start again to be scared until I clip the rope in the next one, so it's all an alternation".

\section{Coping Strategies}

In general, guides consider fear as a normal, healthy, and clever response, since it is a sign of survival instinct. Yet, fear should be kept under control because, when it becomes panic, it may hinder self-perception and focus, making climbers moving hastily or, conversely, preventing action. Usually, when a trainee is afraid, the situation is delicate and there are no codified rules to follow in order to help her. Trainees can react differently to fear: some of them stop climbing, others may aggressively assault the rock wall, and some others withdraw into themselves. Thus, the tension is solved every time in a different way, according to the instructor's mood, the trainee's reaction, and the social situation.

After years of experience, the guides develop a sense of empathy towards trainees that allows them to understand their state of mind and to find the best way to establish contact with them. During our observations, when climbers experienced fear, the guide intervened by talking to them and trying to bring their emotional state back to the necessary calm to make a fair assessment of the wall and to move properly. In these cases, the guide had to choose whether to reassure them or to encourage them; however, in every case the communication was quick and direct. As MG2 affirmed during the interview: "I let her do and I correct her later when she has her feet back on the ground (...). When the climber is hanging there is no room for rational discourses, just reassurance".

Deepening into the topic of reassurance, some of the guides mentioned that physical contact might be a possible effective solution to reassure frightened beginners, which however cannot be pursued, not only because of physical distance, but 
also of social constraints. People may be annoyed or embarrassed by a hand on a shoulder because of cultural and social reasons (e.g. being in front of the whole class). As MG2 stated "You do not reassure somebody with rational reasoning. In every situation, when somebody is afraid you touch her, cuddle her. Of course there are different ways according to different contexts, but anyhow that's what we need [when we are afraid], and that's something that our culture is suspicious about, because touching is allowed only in intimate relationships. "In any case, apart from managing the phenomenon of fear when it occurs, guides do not provide any direct training for the psychological aspects involved. Since fears can be deep inside people, the main worry is not to force beginners to do things they do not feel like doing. Thus, the improvement of the psychological part is left to the individual's commitment; it can be overcome just thanks to inner motivation and will.

\section{Trust}

In climbing, there is a question of objective danger and securing procedures, and a question of subjective perception of both danger and safety that could be referred to as perceived risk and trust. From the interviews with trainees, it emerged that they would take a risk if they think their move will be successful, if they are protected by the gear, and when they trust the belayer. The belayer plays a critical role and the trainees we interviewed were very conscious and felt the responsibility of belaying ("I'm even more stressed when I belay!" T10; "Personally, I feel a lot the responsibility (...), just for the idea that you have the life of someone else in your hands" T5).

The role of the belayer is not confined to managing the rope promptly and properly; she has to be an active observer who makes the climber constantly feel her presence by supporting the climber's actions and encouraging her. Indeed, when engaged in an ascent, the climber has to know that she can rely on the belayer's support ("at the beginning you feel insecure and you have to tell yourself "no, I know that she is holding me', I can trust" T1). When she does not trust the belayer completely, from time to time, she checks whether the other is attentive ("It's annoying when, while belaying, people look around and not at me... I'm not saying that they should always look at me, but I need to know that I'm safe, that they will notice if I fall and that they will try to do something" T8). Trust is a fundamental feeling in the relationship between climber and belayer since it allows the climber to feel safe when moving out of her comfort zone and to have a successful performance.

Thus, it is fundamental for the belayer to learn how to inspire trust by communicating presence and attention, in addition to a correct handling of the rope. In the interviews, the guides told us that they address the issue of trust between climbing partners through specific exercises and simulations. For instance, they propose fall tests to demonstrate the effectiveness of the safety gear and procedures, but also to make trainees realize the importance of the belayer, because, as one of the trainees affirmed "[it is just] by falling that you realize that there is someone holding you" T10. Moreover, the guides propose exercises to let climbers experience the lack of control and their dependence on the belayers, and to train belayers to be precise when communicating, by asking, for example, to climb blindfold just following the belayer's instructions.

\section{Communication}

Communication plays an important role since it is at the basis of the teaching and of the coordination between the climbing partners. Besides the general explanations that are given when everybody is on the ground, the communicative exchanges that occur during the climbs are of particular relevance. In the next paragraph, we report in detail the communication that takes place between instructor and climber and between belayer and climber when the latter is on the wall.

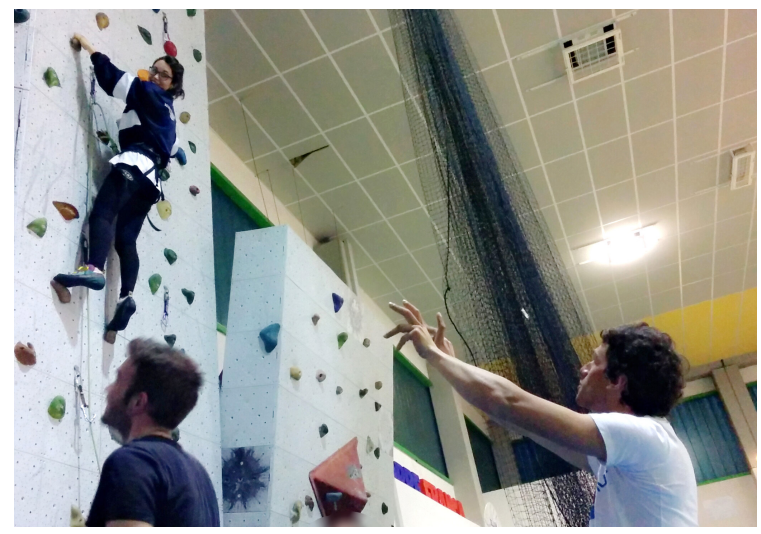

Figure 3. A climbing instructor giving suggestions to a trainee.

\section{Communication between instructor and climber}

During the ascents, the instructor can give suggestions to the belayer about how to handle the friction device, but he follows and gives suggestions especially to the climber (see fig. 3). The instructor can answer a climber's question or spontaneously say something; in any case, the communication occurring while a trainee is hanging on the wall is very brief. The messages towards the climber can take the shape of suggestions (e.g. "try to move your right foot on the green foothold"), instructions (e.g. "outstretch your arms"), reassurances (e.g. "don't worry, you have almost arrived!"), and encouragements ("well done!").

A problem in the communication with climbers that guides have reported is distance and the fact that both climbers and instructors have to yell at each other to be heard ("When the climber is a bit stressed those on the ground start shouting at her 'put your foot there!', but which foot? There where? (...) and then I have to shout and that's terrible, and then inevitably also the belayer starts to shout because she wants to contribute too" MG2). Another reported problem is that sometimes there are misunderstandings caused by the lack of a shared vocabulary. Often trainees do not immediately get 
what the guide is trying to suggest them because they do not know specific climbing terms, or the meaning certain common sentences have in the climbing context, e.g. "leaning back" for getting ready to be lowered.

\section{Communication between climber and belayer}

Normally, during the ascents the climber communicates her needs to the belayer through a series of conventional commands, e.g. "take!" when the climber wants to rest; "slack!" when she needs to clip the rope; "watch me!" when trying a difficult movement; and "lower me!" when, finally, the climber gets to the top. On the other side, the belayer communicates to give suggestions, ask for feedback about the climber's wellbeing, and encourage the climber. Yet, as for the instructors, there can be communication difficulties between climbing partners due to the distance, which can prevent them from seeing and hearing each other (T1 climber - "I turn my head and I tell her, shouting. Even if sometimes it's a bit tiring and I'd prefer her to hear without requiring me to turn my head").

To overcome this problem, usually the climbing partners speak loudly or through gestures like thumbs up when arriving at the top of a climb. As both climber and belayer become more experienced, part of the communication for coordination becomes non-verbal and is conveyed through the rope. When the climber needs to clip the rope in the quickdraws, she can just pull up the rope to signal her need, and an attentive belayer would be ready to give her slack and not to make her tired with several attempts. Especially when the climber is climbing in top rope, the tension of the rope is also used by the belayer to signal presence. By keeping the rope tense and using it to accompany the climber's movements, the belayer communicates that her attention is totally dedicated to the climber. Another interesting aspect that emerged from the interviews with the trainees is that the belayer's emotional state is often a reflection of the climber's one, showing a sense of empathy, which often remains unspoken. The emotions are shared both in negative and positive moments, for example, as T5 expressed, keeping the rope in tension "surely requires focus, and sometimes it is also scary because when one is in trouble there is at least stress", or, as others reported, lowering the climber off evokes satisfaction for the successful climb "I'm enthusiastic for her" T11, "Satisfied for her, for her happiness" T2.

\section{DESIGN SPACE}

Our field study highlighted the main aspects that influence the process of learning how to climb. The findings showed that learning to climb is not just a matter of acquiring new motor skills, but it requires also to build trust with the belayer and to develop mental control over negative emotions. During the learning, positive and negative emotions alternate and the communication between the actors involved plays an important role in facilitating the emergence of the positive ones. The field study allowed us to identify the communication acts exchanged between the instructor and the trainees, and between the climbing partners. All these communication acts are meant to support the climber during her ascent, but in different ways; some aim at supporting the correct execution of movements, while others aim at instilling confidence and building trust. Moreover, we noticed that some communication acts are well defined, while others are very vague and can be conveyed through non-verbal behavior as well (e.g. by handling the rope). In order to have a clear understanding of the differences, we arranged the communication acts along two orthogonal dimensions: a continuum ranging between functionality and emotionality, and a continuum between abstraction and concreteness (fig. 4).

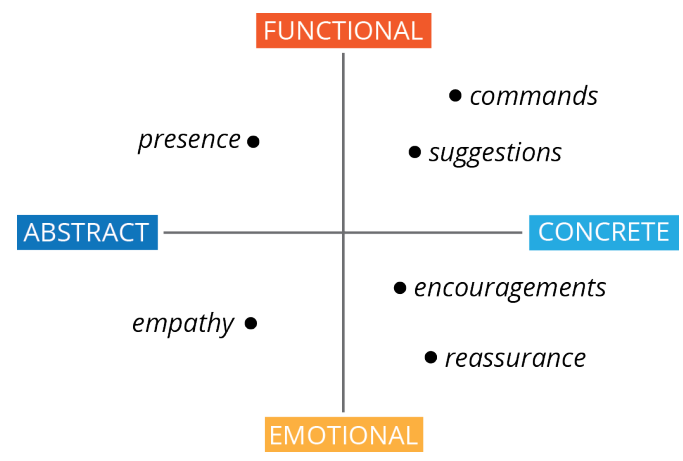

Figure 4. Different communication acts in climbing.

The right upper quadrant defined by the intersection of functional and concrete communication includes commands and suggestions. Commands are meant to support coordination between the climbing partners and are often conveyed through conventional verbal expressions, while suggestions are verbal, defined messages usually aimed at repositioning the climber's body posture or at showing her possibilities, she did not consider. The right lower quadrant defined by the intersection between concrete and emotional communication includes encouragements and reassurance. Both these acts have an emotional purpose, but while reassurance is used when the climber's thrill takes the form of fear hindering her progression, encouragements work as reinforcement of the climber's self-confidence and can be used in many occasions. The quadrants on the abstract side of the continuum contain the communicative acts coveying presence and empathy, which can have both functional and emotional purposes. Presence is the substrate of every communication (i.e. it is expressed every time somebody says something) and can have the purpose of making a climber perform a movement, as well as calming her down. Similarly, empathy can be emotional as well as kinesthetic, the latter referring to people's innate body intelligence about where and when to perform an action in relation to other people [6], and can be useful to anticipate the climbers' needs. However, from our study it emerged that these communicative acts are not always effective since they may suffer from environmental aspects, such as distance and noise. 
These findings suggest that there can be a design space for wearable devices in climbing as communication tools. Wearable devices are small, flexible, unobtrusive devices that are applied in direct contact with the body and work as an augmentation of the body itself by providing information that enhances its native capacities [27]. Wearable devices are suitable for climbing since they would not interpose between the climber and the wall, but would rather work as an enhancement of the climber, thus preserving the direct relationship climbers have with the rock. In this way, the sense of self-challenge and self-efficacy typical of the climbing experience can be maintained. Moreover, wearable devices can support also the need for adventure of climbers, since they can be brought along in every ascent, both indoors and outdoors. Yet, unlike the previous works on wearable devices in climbing, we do not aim at enhancing just the body through the awareness of its performance, but the whole person, considering her body, emotions, and relation to the other actors involved. Specifically, our findings shape a design space for wearable devices that bridge the gap of communication in climbing by providing augmented communication that addresses both the functional and emotional aspects of it. In the following paragraphs, we outline four key design considerations for giving suggestions and managing emotions in climbing using wearable devices.

\section{Key Design Considerations}

\section{Overcome distance and noise}

The distance between the climber and the ground, the environmental noise of gyms, or the wind in outdoor cliffs hamper mutual understanding of the actors involved. They usually shout their instructions, but this is a tiring and not always effective method. These instances suggest an opportunity for the design of wearable devices aimed at augmenting the communication that already exists between instructor and climber, and between belayer and climber. This consideration is further supported by the fact that climbing is generally taught by qualified professional figures, and that successful performances are a matter of interpersonal understanding and trust between the actors involved.

\section{Embrace body and mind}

The evidences of our field study have shown that in climbing the learning of new motor skills and the management of negative emotions are closely intertwined. Some movements generate fear, and fear makes trainees perform poorly (or not at all) some movements. While climbing, communication is used by the instructor and the belayer to maintain or restore calm and focus in the climber, and to exchange practical information about the ascent. The fact that learning to climb does not require only physical training but also a mental one is an important aspect to take into account if the design would explore other forms of communication besides verbal communication. In this latter case, it would be necessary to build a vocabulary of discernible messages that allows the actors involved to express both types of messages, the functional and the emotional ones.
From Fear to Flow

Climbers seek out for positive emotions such as selfefficacy, sense of liberation, and complete focus that this sport can potentially provide, but, prior to that, they need to overcome several negative emotions. Indeed, as discussed by [18], the feeling of flow comes with a certain level of experience, while for beginners it is a state to achieve. Regarding the negative emotions in the learning phase, the guides we interviewed reported that they address fear only when it occurs and when the intensity of the emotion is so high to block the climber; otherwise, it is considered an inherent part of the activity. The potentially worthwhile outcomes of negative emotions (such as anxiety, anger, or tension) in sport are supported also by the literature. For example, Hanin [9] highlights that sometimes negative emotions can help athletes to maintain focus and sustain effort, while Brymer et al. [3] report that, to some extent, climbers even seek for them because without difficulties, there would not be satisfaction. These instances suggest the design of wearable devices aimed at supporting the communication for managing fear, when it occurs with strong intensity. In order to do this, the wearable devices should be activated in real time, so that the instructor can intervene when the trainees are on the wall. However, the aim of these devices should not be that of removing negative emotions, but rather leading beginners towards a state of flow through the feeling of attentiveness and the suggestions of the instructor, by respecting the delicate balance between positive and negative emotions typical of this sport.

\section{Build trust}

Knowing that the belayer is attentive and ready for action is fundamental for the climber's tranquility and performance. However, beginner belayers might not be good yet at reading the signal of stress in climbers and at providing them with practical and mental support. Moreover, from our study it emerged that belayers have feelings of empathy towards the climbers' performance that often remain unspoken, e.g. satisfaction for the successful ascent or tension in a moment of difficulty. Since the climber actually needs to feel the presence and the attention of the belayer, giving her the possibility to express these empathetic feelings could be a way to improve the climber's experience of the ascent. These instances suggest an opportunity for the design of wearable devices that provide the climbing partners with the possibility to express themselves, so that they can attune in joint actions and share their mood.

\section{DISCUSSION}

The design considerations we provided suggest supporting the relational aspect of learning by enhancing interpersonal communication. In this section, we discuss the pros and cons of audio and tactile modalities in augmenting the interpersonal communication that normally occurs between the actors involved. Visual communication will not be considered here since in climbing visual messages would be suitable if displayed on climbing walls [17], but they would not be efficient on a wearable device [13]. On a wearable 
device, changes in a display or in LED lights would not be noticed unless they are integrated with other sound or vibrotactile notifications because the climbers' visual attention is totally engaged in the activity.

\section{Audio Communication}

The expressive richness of audio offers a wide range of opportunities; it is possible to send sound notifications, music, as well as to transmit voice. In sport, sound notifications have been used to give athletes feedback about their performance, e.g. movement sonification has been used in correcting the posture of skiers [10], while by exploiting the rhythm and the tempo of music it is possible to energize the athletes, as the app Nike+ does. Voice has been used by Müller et al. [19] who conveyed spatially distributed audio through headsets to make distant joggers chat and motivate each other. The familiarity people have with speech communication and the ability to express at the same time information and emotionality through intonation and volume make voice communication appropriate for our purposes. Therefore, in order to overcome the problem of distance, voice messages could be conveyed through microphone and earphones, although earphones might cause auditory isolation in the actors, who instead should be aware of the environment, e.g. for noticing people in the nearby or stones falling.

\section{Concept 1: Communication through Helmet}

In order to ensure augmented communication between the actors involved and at the same time not to isolate them from the environment, we envision a helmet augmented with bone conducting earphones and microphone (e.g. Aftershokz Trekz Titanium). All the three actors involved would wear one helmet and communicate through the same channel. In this way, the communication for coordination between climbing partners, as well as the suggestions and the encouragements from the instructor would be covered by voice communication. Therefore, the communication would free and organized through the natural turn taking, as in usual conversations. This very simple concept might solve the issue of distance in climbing quite easily and efficiently, although it does not solve the issues related to the misunderstandings due to the specificity of technical climbing words, and to the time and attention required to process the information.

\section{Haptic Communication}

Haptic feedback is an interesting option since it exploits the peculiarity of wearable devices of being in direct contact with the body. By touching the body, wearable devices can provide cutaneous feedback (such as pressure, heat, or vibration), which is private and direct. The sense of touch is fundamental and already on the alert in climbing. By tackling the wall with bare hands, climbers feel the materiality of the rock in search for the best holds, and through the spatial sensations of touch, like kinesthesis and proprioception, they perceive the inclination of the wall and adjust their balance accordingly [5]. In the literature, haptic feedback has been demonstrated to be valuable for giving motion instructions in the field of sports [25] and to be perceivable also specifically while climbing [13]. Furthermore, haptic feedback has also been investigated as a way to convey emotions [24] and mediated social touch [11] for remote interpersonal interactions, and also for audience cheering up during sport performances [28]. Non-verbal communication through touch is already partially used by climbing partners to communicate needs by pulling the rope or presence by keeping it tensed. Moreover, one of the guides affirmed that, in case of need for reassurance, it would be of great help to touch the frightened person, but this cannot be done not to cause social embarrassment. In this case, mediated social touch could be a solution worth exploring.

\section{Concept 2: Vibrotactile Bracelets and T-Shirt}

Given the aforementioned qualities, we think haptics to be a modality worth exploring for the augmentation of both functional and emotional communication in climbing. Suggestions about movement could refer to directions or body posture and might regard every limb of the climber; hence, in order to make her understand which part of the body to move and how, the messages should be directed on specific body parts. Considering the case of suggesting directions, the climber could wear four bracelets enhanced with a row of three vibrotactile motors on each wrist and ankle. The vibrotactile motors could vibrate sequentially in order to signify "move right" or "move left". In this way, by exploiting body location and form factor of the wearable device, it would be possible to convey functional messages such as directions. Conversely, emotional messages, which are not related to a specific body part, could be conveyed by a row of vibrotactile motors located on the back of the Tshirt, between the shoulder blades, so that vibrating motors not hamper the movements of the climber. By making the motors vibrate sequentially and modulating the vibration with different levels of intensity, speed and number of repetitions, it could be possible to express different messages. For example, a soft and slow vibration could mean reassurance, while a more intense and fast one could mean encouragement. Such augmented T-shirt could help the climber feel the attention and presence of the instructor or the belayer, according to whom is sending the vibrations. Instructors could send the haptic messages through a tablet, while belayers through a pedalboard, since their hands are already busy managing the rope.

\section{LIMITATIONS AND FUTURE WORK}

The field study presented in this paper has some limitations. The number of participants was limited and the study has been conducted in a defined geographical area with a specific culture. Thus, there is no evidence that our results can be extended to climbers from other regions. Furthermore, the concepts we proposed are illustrative and do not aim to be exhaustive. We chose to present the advantages and disadvantages of different modalities separately, but as the example of visual communication showed, multimodality could be a way to strengthen the effectiveness of augmented communication and it is an option worth exploring. Future 
work will include the iterative involvement of climbers in the design process in order to validate our concepts and prototypes of wearable devices.

\section{CONCLUSION}

Designing a wearable technology for an extreme sport raises great challenges about what kind of support it could provide, and when and how it should intervene. From our field study, it emerged that the learning difficulties of beginner climbers are due to a high level of involvement of negative emotions such as discomfort and fear. The fear beginners experience is mostly caused by the novelty of moving along a vertical wall and by depending on another person for safety. Grounding on these findings, we discussed four considerations that define the design space of wearable devices as means for augmented communication between the climber, the belayer, and the instructor. These considerations suggest a design that does not aim at removing completely the negative emotions from climbing, since they are peculiar to this sport. Conversely, the design should be meant to support learning while maintaining the delicate balance between positive and negative emotions in this sport.

\section{REFERENCES}

1. Bächlin, M., Förster, K., and Tröster, G. SwimMaster: a wearable assistant for swimmer. Proc. of Ubicomp'09, (2009).

2. Beyer, H. and Holtzblatt, K. Contextual design: defining customer-centered systems. Elsevier, 1997.

3. Brymer, E. and Schweitzer, R. Extreme sports are good for your health: A phenomenological understanding of fear and anxiety in extreme sport. Journal of Health Psychology, (2012).

4. Daiber, F., Kosmalla, F., Wiehr, F., and Krüger, A. Outdoor Nature Lovers vs. Indoor Training Enthusiasts: A Survey of Technology Acceptance of Climbers. Proc. of CHI'16, (2016).

5. Dutkiewicz, J. Pretzel Logic: An Embodied Ethnography of a Rock Climb. Space and Culture 18, (2014), 25-38.

6. Fogtmann, M.H., Fritsch, J., and Kortbek, K.J. Kinesthetic Interaction - Revealing the Bodily Potential in Interaction Design. Proceedings of $\mathrm{OzCHI}^{\prime} 08$, (2008).

7. Gardner, T. Popularity and economic benefit of mountaineering: instant expert. BMC Working for climbers, hill walkers \& mountaineers, 2015. https://www.thebmc.co.uk/participation-in-climbingmountaineering.

8. Glaser, B.G. and Strauss, A.L. The discovery of grounded theory: Strategies for qualitative research. Transaction Publishers, 2009.

9. Hanin, Y.L. Emotions in Sport: Current Issues and Perspectives. In G. Tenenbaum and R.C. Eklund, eds., Handbook of Sport Psychology, 2007, 31-58.

10. Hasegawa, S., Ishijima, S., Kato, F., Mitake, H., and Sato, M. Realtime Sonification of the Center of Gravity for Skiing. Proc. of $A H^{\prime} 12$, ACM Press (2012), 3-6.

11. Huisman, G., Darriba Frederiks, A., Van Dijk, B., Hevlen, D., and Krose, B. The TaSSt: Tactile sleeve for social touch. World Haptics Conference (2013).

12. Kosmalla, F., Daiber, F., and Krüger, A. ClimbSense Automatic Climbing Route Recognition using Wristworn Inertia Measurement Units. Proc. CHI'15, ACM Press (2015), 2033-2042.

13.Kosmalla, F., Wiehr, F., Daiber, F., Krüger, A., and Löchtefeld, M. ClimbAware - Investigating Perception and Acceptance of Wearables in Rock Climbing. Proceedings of CHI'16, ACM Press (2016).

14. Ladha, C., Hammerla, N.Y., Olivier, P., and Plötz, T. ClimbAX: Skill Assessment for Climbing Enthusiasts. Proceedings of Ubicomp'13, (2013), 235-244.

15. Levenhagen, M. "A Stage Model of Why Climbers Climb And How It Frames the Discussions of Recent Climbing Controversies." Journal of Mountaineering, (2010).

16. Lewis, N. The Climbing Body, Nature and the Experience of Modernity. Journal of Body and Society 6, 10 (2000), 58-80.

17.Liljedahl, M., Lindberg, S., and Berg, J. Digiwall - an Interactive Climbing Wall. Proc. of $A C E^{\prime} 05$, ACM Press (2005), 225-228.

18. MacAloon, J., Csikszentmihalyi, M., Harris, J.C., and Park, R.J. Deep Play and the Flow Experience in Rock Climbing. In J.C. Harris and R.J. Park, eds., Play, games and sports in cultural contexts. 1983, 361-384.

19. Müller, F.F., O’Brien, S., and Thorogood, A. Jogging over a Distance. Proc. of CHI'07, ACM Press (2007).

20. Nguyen, L.N.N., Rodríguez-Martín, D., Català, A., Pérez-López, C., Samà, A., and Cavallaro, A. Basketball Activity Recognition using Wearable Inertial Measurement Units. Proc. of Interacción'15, (2015).

21.Schmid, A., Kleemann, M., Merritt, T., and Selker, T. Tactile Communication in Extreme Contexts: Exploring the Design Space through Kiteboarding. Proc. of INTERACT '15, (2015), 37-54.

22. Schmidt, R.A. and Lee, T.D. Motor Control and Learning. Human Kinetics, 2011.

23. Schöning, J., Panov, I., and Kessler, C. No vertical limit - Conceptual LBS design for climbers. Ext. Abstracts CHI 2007, ACM Press (2007), 1-5.

24. Smith, J. and MacLean, K.E. Communicating emotion through a haptic link: Design space and methodology. International Journal of Human-Computer Studies 65, 4 (2007), 376-387.

25. Spelmezan, D. An investigation into the use of tactile instructions in snowboarding. Proc. of MobileHCI'12, ACM Press (2012).

26. Vallerand, R.J. and Blanchard, C.M. The study of emotion in sport and exercise. Historical, definitional, and conceptual perspectives. In Y.L. Hanin, ed., Emotions in Sport. Human Kinethics, 2000, 3-37.

27. Viseu, A. and Suchman, L. Wearable Augmentations: Imaginaries for the Informed Body. In Technologized Images, Technologiezed Bodies, 2010, 161-184.

28. Woźniak, P., Knaving, K., Björk, S., and Fjeld, M. RUFUS: Remote Supporter Feedback for Long-Distance Runners. Proc. of MobileHCI'15, ACM Press (2015). 